\title{
ARMAZENAMENTO DE PESSSGOS (Prunus persica L.), CULTIVAR CHIRIPÁ, EM ATMOSFERA CONTROLADA
}

\author{
STORAGE OF CHIRIPA PEACH (Prunus persica L.) IN CONTROLLED ATMOSPHERE
}

\author{
Cesar Valmor Rombaldi ${ }^{1}$ Jorge Adolfo Silva ${ }^{2}$ Aguinaldo Parussolo $^{3}$ Luciano Lucchetta ${ }^{4}$ \\ Márcio Roggia Zanuzo ${ }^{4}$ César Luis Girardi ${ }^{5}$ Ruffino Fernando Flores Cantillano ${ }^{6}$
}

RESUMO

Uma das principais alterações durante o armazenamento refrigerado de pêssegos é a ocorrência de lanosidade. No presente trabalho, foi estudado o efeito da atmosfera controlada sobre a conservação de pêssegos da cultivar Chiripá e, em particular, no controle de lanosidade. Os pêssegos foram colhidos com valores médios de firmeza de polpa

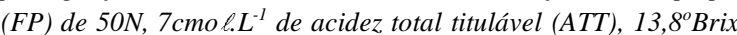
de sólidos solúveis totais (SST) e coloração de fundo verdeesbranquiçada. As frutas foram armazenadas em dois sistemas: 1) ar refrigerado (AR) a $0^{\circ} \mathrm{C} \pm 0,5^{\circ} \mathrm{C}$ e $90 \pm 5 \%$ de umidade relativa (UR); 2) atmosfera controlada (AC) a $0^{\circ} \mathrm{C} \pm 0,5^{\circ} \mathrm{C}$ e $95 \pm 2 \% U R$, 1,5 $\mathrm{KPa}$ de $\mathrm{O}_{2}$ e $5 \mathrm{KPa}$ de $\mathrm{CO}_{2}$. $\mathrm{Na}$ instalação do experimento, aos 30 dias e aos 45 dias, coletaram-se amostras para avaliações de FP, SST, ATT, ocorrência de lanosidade e de escurecimento interno, e análise sensorial. Estas análises foram realizadas 24 horas e 72 horas após a retirada das frutas das câmaras frias, em cujo período foram mantidas a $20^{\circ} \mathrm{C} \pm 2{ }^{\circ} \mathrm{C}$. A AC foi eficiente no controle de lanosidade, mantendo os pêssegos da cv. Chiripá em condições de comercialização por, no mínimo, 45 dias. Em AR este período foi inferior a 30 dias e ocorreram perdas significativas na qualidade das frutas.

Palavras-chave: maturação, lanosidade.

\section{SUMMARY}

The woolly breakdown is one of the major disorders during cold storage of "Chiripá" peaches. In the present work the effects of controlled atmosphere (CA) on peaches storability were studied, particularly in the control of woolliness. The peaches were harvested with 50N of pulp firmness (PF),

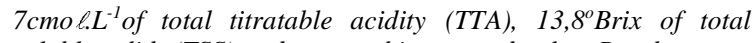
soluble solids (TSS) and green-white ground color. Peaches were stored in two systems: 1) refrigerated air storage (RA) at $O^{\circ} \mathrm{C} \pm 0,5^{\circ} \mathrm{C}$ and $90 \pm 5 \%$ of relative humidity $(R U)$ or 2) controlled atmosphere (CA) at $0^{\circ} \mathrm{C} \pm 0,5^{\circ} \mathrm{C}, 95 \pm 2 \%$ of $\mathrm{RU}, 1,5 \mathrm{KPa}$ of $\mathrm{O}_{2}$ and $5 \mathrm{kPa}$ de $\mathrm{CO}_{2}$. At the installation of the experiment, 30 days after and 45 days after, samples were collected to evaluate PF, TTA, TSS, woolly breakdown, browning and for organoleptic analysis. These evaluations were done 24 and 72 hours after sampling. The results showed that $C A$ is capable of reducing efficiently the woolliness and maintaining fruit quality for 45 days of storage. In $R A$ this period was less than 30 days with significative losses in the quality.

Key words: ripening, woolliness.

\section{INTRODUÇÃO}

A cultivar chiripá, de pêssego para consumo in natura, criada pela EMBRAPA - Clima Temperado - Pelotas, é a mais plantada no Sul do Brasil. Ela produz frutas de tamanho médio a grandes, com cerca de $190 \mathrm{~g}$, polpa branca, alto potencial de acúmulo de açúcares, caroço aderente e epiderme com coloração de superfície avermelhada e cor de fundo esverdeada. Na maioria das regiões, a colheita desta cultivar é realizada no período de 15 de dezembro a 15 de janeiro (MEDEIROS \& RASEIRA, 1998).

\footnotetext{
${ }^{1}$ Engenheiro Agrônomo, PhD., Professor Adjunto do Departamento de Ciência e Tecnologia Agroindustrial (DCTA), Universidade Federal de Pelotas (UFPEL), CP 354, 96010-900 Pelotas, RS. E-mail: cesarvrf@ufpel.tche.br. Autor para correspondência.

${ }^{2}$ Engenherio Agrônomo, DSc., Pesquisador DCTA-UFPEL. E-mail: ctajorge@ufpel.tche.br.

${ }^{3}$ Químico Industrial de Alimentos, aluno do Programa de Mestrado em Ciência e Tecnologia Agroindustrial (PMCTA), DCTA-UFPEL. E-mail: aguipar@ufpel.tche.br.

${ }^{4}$ Acadêmico de Agronomia, Bolsista de Iniciação Científica do CNPq. E-mail: luccheta @ufpel.tche.br.

${ }^{5}$ Engenheiro Agrônomo, MSc. Pesquisador do CNPUV-EMBRAPA, Bento Gonçalves, RS. E-mail: girardi@cnpuv.embrapa.br.

${ }^{6}$ Engenheiro Agrônomo, PhD. Pesquisador do CPACT-EMBRAPA, Pelotas, RS.
} 
Para prolongar o período de armazenamento, os pêssegos desta cultivar vêm sendo colhidos em estádios iniciais de maturação, com firmeza de polpa acima de $65 \mathrm{~N}$, acidez total titulável entre 8 e $10 \mathrm{cmo} \ell . \mathrm{L}^{-1}$, sólidos solúveis totais entre 12,5 e $13,5^{0}$ Brix e coloração de fundo verdeopaca. Em conseqüência, a qualidade sensorial destas frutas é baixa e a suscetibilidade à ocorrência de lanosidade aumenta. Para aumentar a qualidade organoléptica destes pêssegos, a colheita deve ser realizada quando as frutas atingem uma coloração de fundo verde-esbranquiçada, 45 a $60 \mathrm{~N}$ de firmeza de polpa, 13,5 a $14,5^{0}$ Brix de sólidos solúveis, e 6 a $8 \mathrm{cmo} \ell . \mathrm{L}^{-1} \mathrm{de}$ acidez total titulável. No entanto, este procedimento diminui o período de armazenamento refrigerado (AR). Já a colheita tardia, quando os pessegos têm em torno de 20 a $30 \mathrm{~N}$ de firmeza, de 14 a $15^{\circ}$ Brix e acidez total titulável inferior a $6 \mathrm{cmo} \ell . \mathrm{L}^{-1}$, resulta em pêssegos com elevada qualidade, mas com baixo potencial de conservação, sendo mais apropriados para o consumo imediato.

No Brasil, o sistema mais empregado para a conservação de pêssegos in natura é o armazenamento em ar refrigerado. Neste sistema, o período seguro de estocagem de pêssegos varia de 5 a 40 dias, dependendo da cultivar, do ponto de colheita, da região de cultivo e da qualidade das câmaras frias, conforme observações feitas por MEREDITH et al. (1989), TAYLOR $\boldsymbol{e t}$ al.(1994) e SALVADOR $\boldsymbol{e t}$ al. (1998). A perda de firmeza da polpa (BEN-ARIE \& SONEGO, 1980; MANESS et al., 1993; SONEGO $\boldsymbol{e t}$ al., 1994), a ocorrência de distúrbios fisiológicos, especialmente lanosidade e escurecimento interno (ROBERTSON et al., 1992; LURIE, 1993; LUCHSINGER $\boldsymbol{e t}$ al., 1996) e podridões (GOTTINARI et al., 1998; CERETA, 1999) têm sido relatadas como as principais alterações indesejáveis durante o armazenamento em AR.

Outras alternativas, como o emprego de atmosferas modificadas (LURIE, 1993), aquecimentos intermitentes e choques de $\mathrm{CO}_{2}$ (TONUTTI et al., 1998), têm sido testados para reduzir estes problemas. Os resultados são variados, indicando, em alguns casos, limitações econômicas e falta de reprodutibilidade conforme MITCHELL \& CRISOSTO (1995).

O emprego de atmosfera controlada (AC) é citado como o sistema mais eficiente para a estocagem de frutas de caroço, embora os custos de instalação e operacionais sejam maiores do que o armazenamento em ar refrigerado (AR) conforme LURIE (1992) e PALMER et al. (1997). Comparadas com as pomáceas, as frutas de caroço toleram teores de $\mathrm{CO}_{2}$ mais elevados durante o armazenamento em AC. Assim, por exemplo, para a maioria das cultivares de maçã, a concentração deste gás deve ser mantida entre $0,5 \mathrm{KPa}$ (Fuji) e 4,0KPa (Golden Delicious) observado por BRACKMANN \& SAQUET (1995). Para a maioria das frutas de caroço estas concentrações podem ser aumentadas para até 10KPa conforme LURIE (1992) e CERETA (1999), sem a ocorrência de distúrbios ocasionados pelo $\mathrm{CO}_{2}$. Algumas cultivares de cereja toleram teores de $\mathrm{CO}_{2}$ de até $20 \mathrm{KPa}$ (PECH et al., 1994). As concentrações de $\mathrm{O}_{2}$ normalmente empregadas variam de $1 \mathrm{KPa}$ a $3 \mathrm{KPa}$, observando-se maior eficiência do sistema na medida que a concentração de $\mathrm{O}_{2}$ diminui, de acordo com LURIE (1992) e PALMER et al. (1997).

No Brasil, os primeiros experimentos com o armazenamento de pêssegos em atmosfera controlada foram realizados no Núcleo de PósColheita da UFSM (CERETA, 1999). Os resultados obtidos com pêssegos da cultivar Eldorado indicaram que este sistema permite diminuir a ocorrência de escurecimento interno e de podridões.

Dentro deste contexto, buscou-se estudar o efeito da AC na manutenção da qualidade de pêssegos da cultivar Chiripá, utilizando-se o armazenamento em AR como tratamento controle.

\section{MATERIAL E MÉTODOS}

Foram utilizados pêssegos da cultivar Chiripá colhidos em 24/12/1998 em pomares comerciais no Município de Campestre da Serra, Região Nordeste do Estado do Rio Grande do Sul, apresentando coloração de fundo verde-esbranquiçado, firmeza de polpa (FP) de $50 \mathrm{~N}$, sólidos solúveis totais (SST) de $13,8^{\circ}$ Brix e $7 \mathrm{cmo} \ell . \mathrm{L}^{-1}$ de acidez total titulável (ATT). As análises laboratoriais foram conduzidas nos Laboratórios do Departamento de Ciência e Tecnologia Agroindustrial da Faculdade de Agronomia Eliseu Maciel da Universidade Federal de Pelotas.

Os pêssegos foram pré-resfriados com ar forçado, acondicionados em caixas plásticas de $20 \mathrm{Kg}$ e mantidos em armazenamento refrigerado (AR) em câmara fria comercial a $0^{\circ} \mathrm{C} \pm 0,5^{\circ} \mathrm{C}$ e $90 \pm 5 \%$ de umidade relativa (UR) e em câmara de atmosfera controlada (AC) comercial. Para obtenção da $\mathrm{AC}$, inicialmente, injetou-se $\mathrm{N}_{2}$ até que a concentração de $\mathrm{O}_{2}$ atingisse $1,5 \mathrm{KPa}$. Em seguida, ajustou-se a concentração de $\mathrm{CO}_{2}$ para $5 \mathrm{KPa}$ pela injeção deste gás, proveniente de cilindro com concentração de $99,5 \%$ de $\mathrm{CO}_{2}$. As condições de AC foram obtidas 24 horas após o fechamento da câmara. A temperatura média da polpa das frutas, medida com termômetro de mercúrio, foi de $0,0^{\circ} \mathrm{C} \pm 0,5^{\circ} \mathrm{C}$. 
$\mathrm{Na}$ instalação do experimento, aos 30 dias e aos 45 dias de armazenamento, foram coletadas 20 amostras com 20 pêssegos cada, de cada sistema de refrigeração (AR e AC). Dessas 20 amostras, dez foram avaliadas 24 horas após a retirada da câmara frigorífica e dez foram avaliadas 72 horas após a retirada da câmara, simulando um período de comercialização. As análises realizadas foram perda de peso, FP, ATT, SST, ocorrência de lanosidade e/ou de escurecimento interno, e realizou-se análise sensorial, na qual foram avaliados os seguintes atributos: aparência externa, odor, textura, sabor e qualidade geral.

A perda de peso, a FP, a ATT e os SST foram determinados de acordo com a metodologia descrita por GOTTINARI et al. (1998) e CERETA (1999). Para a avaliação da FP, foi utilizado um penetrômetro de mão com ponteira de $8 \mathrm{~mm}$ e os resultados expressos em Newton (N). Para a determinação de ATT e SST, foi realizada a extração, em centrífuga, do suco de dez frutas. A ATT foi medida através de titulometria de neutralização com $\mathrm{NaOH} \quad 0,1 \mathrm{~N}$, utilizando fenolftaleína como indicador e os resultados foram expressos em cmo $\ell . \mathrm{L}^{-1}$. SST foram determinados com o auxílio de um refratômetro de bancada. Foi avaliada visualmente a ocorrência de lanosidade e de escurecimento interno do mesocarpo em 10 frutas de cada amostra, expressando-se os resultados em percentual. A avaliação sensorial foi realizada por um grupo de 10 degustadores não treinados, adotando-se uma escala crescente de preferência onde a nota 0 correspondia a frutas de péssima qualidade e a nota 9, a frutas de excelente qualidade.

Foram consideradas aptas à comercialização as frutas que atendiam, no conjunto: FP acima de $20 \mathrm{~N}$, ocorrência de lanosidade e de escurecimento inferiores a $20 \%$ e nota acima de 5 na avaliação sensorial (SONEGO et al., 1994). delineamento inteiramente casualizado, com dez repetições. Os dados obtidos foram submetidos à análise de variância e, para comparação de médias, ao teste de Duncan, em nível de $5 \%$ de probabilidade de erro. As variáveis expressas em percentagem foram transformadas para arcoseno.

\section{RESULTADOS E DISCUSSÃO}

Em AR, a firmeza de polpa diminuiu significativamente, de $50 \mathrm{~N}$ para $18 \mathrm{~N}$, em 30 dias de

- Não detectado. armazenamento refrigerado, mais 24 horas a $20^{\circ} \mathrm{C} \pm 2^{\circ} \mathrm{C}$. Em AC esta diminuição foi significativamente inferior, de $50 \mathrm{~N}$ para $40 \mathrm{~N}$, no mesmo período. Quando os pêssegos foram analisados 72 horas após a retirada das câmaras frias, observou-se uma menor diminuição da FP das frutas em AR, passando de $50 \mathrm{~N}$ para $34 \mathrm{~N}$. Esse comportamento pode ser explicado pela elevada incidência de lanosidade (100\%) nos pêssegos armazenados em AR, superestimando os valores de FP. Em AC, a FP reduziu de $50 \mathrm{~N}$ para $30 \mathrm{~N}$ (Tabela 1). Este comportamento está de acordo com o observado anteriormente por TONUTTI $\boldsymbol{e t}$ al. (1998) que verificaram uma redução da incidência de lanosidade em pêssegos armazenados em baixas concentrações de $\mathrm{O}_{2}$ e/ou altas de $\mathrm{CO}_{2}$. Após 45 dias de armazenamento refrigerado, também houve redução da firmeza de polpa dos pêssegos, tanto em AR como em AC porém quando se comparam os períodos após a retirada das câmara frigorífica em $\mathrm{AR}$, não houve variação significativa da FP. Isto pode ser devido a já elevada lanosidade detectada no período de 24 horas (35\% das frutas). Para aquelas frutas armazenadas em AC, a FP diminuiu, passando de 35 para $25 \mathrm{~N}$. Esta alteração é coerente com a evolução da maturação e com os baixos percentuais de lanosidade encontrado nestas frutas (entre 2 e $8 \%)$.

O teor de SST aumentou durante os primeiros 30 dias de armazenamento em AR (mais $24 \mathrm{~h}$ a $20 \pm 2^{\circ} \mathrm{C}$ ), passando de $13,8^{\circ}$ Brix para $14,2^{\circ}$ Brix. No período de 24 até 72 horas após a retirada das câmaras frias, somente as frutas em AC apresentaram um incremento significativo, no teor

Tabela 1 - Avaliação físico-química, fisiológica e sensorial de pêssegos, cv. Chiripá, após 30 dias de armazenamento refrigerado e em atmosfera controlada, Pelotas 1998 .

\begin{tabular}{|c|c|c|c|c|c|}
\hline \multirow{3}{*}{$\begin{array}{l}\text { Avaliações } \\
\text { Firmeza de polpa (N) }\end{array}$} & \multirow{3}{*}{$\begin{array}{l}\text { Valores } \\
\text { iniciais } \\
50 \mathrm{a} * *\end{array}$} & \multicolumn{2}{|c|}{$\begin{array}{l}\text { Armazenamento } \\
\text { refrigerado }\end{array}$} & \multicolumn{2}{|c|}{$\begin{array}{l}\text { Atmosfera } \\
\text { controlada }\end{array}$} \\
\hline & & $24 h^{*}$ & $72 \mathrm{~h} *$ & $24 h^{*}$ & $72 \mathrm{~h} *$ \\
\hline & & $18 \mathrm{~d}$ & 34 & 40 & 30 \\
\hline Sólidos solúveis totais $\left({ }^{\circ} \mathrm{Brix}\right)$ & $13,8 \mathrm{c}$ & $14,2 \mathrm{~b}$ & $14,3 \mathrm{~b}$ & $14,0 \mathrm{bc}$ & $15,3 \mathrm{a}$ \\
\hline Acidez total titulável $\left(\mathrm{cmol} . \mathrm{L}^{-1}\right)$ & $7 \mathrm{~b}$ & $7 \mathrm{~b}$ & $7,5 \mathrm{ab}$ & $7,5 \mathrm{ab}$ & $6,0 \mathrm{c}$ \\
\hline Lanosidade $(\%)$ & - & $8 \mathrm{~b}$ & $100 \mathrm{a}$ & - & - \\
\hline Escurecimento (\%) & - & $12 \mathrm{~b}$ & $25 \mathrm{a}$ & - & - \\
\hline Nota $(0$ a 9$)$ & $7 \mathrm{a}$ & $4 \mathrm{~b}$ & $2 \quad \mathrm{c}$ & $6 \mathrm{a}$ & $7 \mathrm{a}$ \\
\hline Perda de peso $(\%)$ & & $2 \mathrm{a}$ & 3 a & $2,5 \mathrm{a}$ & 3 a \\
\hline
\end{tabular}

* Avaliações realizadas 24 horas e 72 horas após a retirada das frutas da câmara fria, mantidas a $20 \pm 2^{\circ} \mathrm{C}$;

** Médias não seguidas pela mesma letra na mesma linha, diferem entre si pelo teste de Duncan em nível de 5\% de probabilidade de erro. CVs AR 24/72h: $\mathrm{FP}=3.2 / 4,7$, SST $=2,9 / 4,7$, ATT 8,6/6,2, Lanosidade $=5,4 / 1,5$, Escurecimento=5,2/6,4, Análise Sensorial=17,8/16,1, Perda de Peso: 3,5/3,7. CVs AC 24/72h: $F P=4,2 / 3,1, \mathrm{SST}=3,7 / 3,3$, ATT 6,5/7,0, Análise Sensorial=15,4/16,1, Perda de Peso: 4,1/3,2. 
de SST, atingindo $15,3^{\circ}$ Brix. PRATELLA et al. (1988), SOUTY et al. (1990) e MURRAY \& VALENTINI (1998) observaram que o potencial de incremento de SST após a colheita de frutas de caroço está diretamente relacionado com o ponto de colheita e a qualidade do sistema de armazenamento. Isto explica o comportamento dos pêssegos armazenados em AC. As condições de estocagem permitiram reduzir a velocidade das reações metabólicas e preservar as reservas metabólicas. Os pêssegos mantidos em AR após 45 dias, entretanto, não tiveram suas reservas suficientemente protegidas e apresentaram um decréscimo significativo de SST.

As maiores variações de ATT durante os primeiros 30 dias de armazenamento foram observadas nos pêssegos em AC, 72 horas após a retirada das frutas da câmara fria, diminuindo de 7 para $6 \mathrm{cmo} \ell . \mathrm{L}^{-1}$. LURIE (1992) e ROBERTSON $\boldsymbol{e}$ al. (1992) também determinaram que pêssegos armazenados em AC apresentam maiores perdas de acidez após a retirada das câmaras frias do que aqueles mantidos em AR. Os autores citam que isto se deve à maior integridade biológica das frutas armazenadas em AC.

Em AR, a ocorrência de lanosidade aumentou durante o armazenamento, atingindo $8 \%$ das frutas após 30 dias (mais $24 \mathrm{~h}$ a $20 \pm 2^{\circ} \mathrm{C}$ ). A manutenção dos pêssegos durante 72 horas a $20 \pm 2^{\circ} \mathrm{C}$ aumentou ainda mais este distúrbio atingindo $100 \%$ das frutas. Em AC, não se detectou a ocorrência de lanosidade nos 30 primeiros dias, nem mesmo após $72 \mathrm{~h}$ a $20 \pm 2^{\circ} \mathrm{C}$. Segundo MITCHELL \& CRISOSTO (1995), este distúrbio fisiológico é dependente da regulação da atividade de enzimas envolvidas no metabolismo da parede celular. Quando as condições de armazenamento favorecem a atividade de pectil-metil-esterases e exopoligalacturonases, em detrimento às endopoligalacturonases, aumenta a incidência de lanosidade. LURIE (1992), SONEGO et al. (1994), PALMER et al. (1997), e TONUTTI et al (1998) citam que o armazenamento em AC reduz eficientemente a atividade destas enzimas, diminuindo a incidência de lanosidade, o que o que pode explicar os resultados desta experiência. PRESSEY \& AVANTS (1978) ressaltam que os efeitos positivos da AC são mais marcantes em pêssegos de polpa branca e de caroço molar, nos quais a suscetibirepresentada pelas baixas notas abaixo de 2 obtidas na avaliação sensorial. Em AC, também houve perda na lidade à lanosidade é maior.

Até 30 dias de avaliação, o escurecimento interno só foi observado em AR. Nestas condições os valores foram elevados, atingindo $12 \%$ dos pêssegos após $24 \mathrm{~h}$ a $20 \pm 2^{\circ} \mathrm{C}$, aumentando para $25 \%$ após 72 horas a $20^{\circ} \mathrm{C} \pm 2^{\circ} \mathrm{C}$. Assim, o aumento do período de exposição da fruta à temperatura ambiente propicia a manifestação desta alteração fisiológica. Por outro lado, estes resultados demonstram que o aumento da concentração de $\mathrm{CO}_{2}$ para $5 \mathrm{KPa}$ e a redução do $\mathrm{O}_{2}$ para $1,5 \mathrm{KPa}$, evita a ocorrência deste distúrbio.

A perda média de peso durante $\mathrm{O}$ armazenamento em AR e AC, após 24 e $72 \mathrm{~h}$ a $20 \pm 2^{\circ} \mathrm{C}$, foi baixa, mantendo-se em valores entre 2 e $3 \%$. Comparados com os dados por MITCHELL \& CRISOSTO (1995), GOTTINARI et al. (1998) e CERETA (1999), que determinaram percentagens entre 3 e $7 \%$, estes valores podem ser considerados bons.

A análise conjunta dos dados obtidos nestes primeiros 30 dias de estocagem indica que somente os pêssegos da cv. Chiripá armazenados em $\mathrm{AC}$ atendem os critérios pré-determinados para a comercialização. Os pêssegos mantidos em AR apresentaram alta incidência de frutas com lanosidade (100\%) e escurecimento (25\%), e notas na análise sensorial inferiores a 5 .

O prolongamento do período de avaliação até 45 dias (Tabela 2) em AR aumentou significativamente ocorrência de lanosidade e de escurecimento dos pêssegos, atingindo de 25 a $100 \%$ das frutas. Além disso, houve uma marcante redução na qualidade organoléptica das frutas,

Tabela 2 - Avaliação físico-química, fisiológica e sensorial de pêssegos, cv. Chiripá, após 45 dias de armazenamento refrigerado e em atmosfera controlada, Pelotas 1998.

\begin{tabular}{|c|c|c|c|c|c|}
\hline \multirow{3}{*}{$\begin{array}{l}\text { Avaliações } \\
\text { Firmeza de polpa (N) }\end{array}$} & \multirow{3}{*}{$\begin{array}{l}\text { Valores } \\
\text { iniciais } \\
50 \mathrm{a}^{* *}\end{array}$} & \multicolumn{2}{|c|}{$\begin{array}{l}\text { Armazenamento } \\
\text { refrigerado }\end{array}$} & \multicolumn{2}{|c|}{$\begin{array}{l}\text { Atmosfera } \\
\text { controlada }\end{array}$} \\
\hline & & \multirow{2}{*}{$\frac{24 \mathrm{~h}^{*}}{28 \mathrm{~b}}$} & $72 \mathrm{~h}^{*}$ & $24 \mathrm{~h} *$ & $72 \mathrm{~h} *$ \\
\hline & & & 25 & $35 \mathrm{c}$ & 25 \\
\hline Sólidos solúveis totais $\left({ }^{\circ}\right.$ Brix) & $13,8 \mathrm{c}$ & $13 \mathrm{c}$ & 13 & $14 \mathrm{~b}$ & $15 \mathrm{a}$ \\
\hline Acidez total titulável $\left(\mathrm{cmol} \cdot \mathrm{L}^{-1}\right)$ & $7 \mathrm{~b}$ & $8 \mathrm{~b}$ & $9 \mathrm{ab}$ & $7 \mathrm{~b}$ & $6,0 \quad \mathrm{c}$ \\
\hline Lanosidade $(\%)$ & - & $35 \mathrm{~b}$ & $100 \mathrm{a}$ & $8 \mathrm{c}$ & 2 \\
\hline Escurecimento (\%) & - & $25 \mathrm{~b}$ & 55 a & $5 \mathrm{~d}$ & 8 \\
\hline Nota (0 a 9) & 7 a & $2 \mathrm{~b}$ & 0 & $6 a$ & 5 a \\
\hline Perda de peso $(\%)$ & & $3 a$ & $3,5 \mathrm{a}$ & $3 a$ & $3,5 \mathrm{a}$ \\
\hline
\end{tabular}

* Avaliações realizadas 24 horas e 72 horas após a retirada das frutas da câmara fria, mantidas a $20 \pm 2^{\circ} \mathrm{C}$;

** Médias não seguidas pela mesma letra na mesma linha, diferem entre si pelo teste de Duncan em nível de 5\% de probabilidade de erro. CVs AR 24/72h: FP=2,4/2,9, SST $=3,7 / 4,2$, ATT 6,4/5,5, Lanosidade $=3,3 / 1,2$, Escurecimento=5,6/7,8, Análise Sensorial=16,5/14,3, Perda de Peso: 2,5/3,2. CVs AC 24/72h: FP=3,5/2,6, SST=2,7/2,9, ATT 4,8/5,3, Lanosidade=3,8/1,0, Escurecimento=4,5/3,2, Análise Sensorial=14,3/12,4, Perda de Peso: 2,2/2,4.

- Não detectado.

Ciência Rural, v. 32, n. 1, 2002. 
qualidade, mas foram mantidas as condições mínimas para a comercialização: FP acima de $20 \mathrm{~N}$, distúrbios fisiológicos abaixo de $20 \%$ e as notas de análise sensorial acima de 5 .

Em AR, as notas da análise sensorial reduziram significativamente, tanto na avaliação realizada após 30 dias quanto após 45 dias de frigoconservação, reduzindo de valores médios iniciais de 7, para menor do que 4. Em AC, não houve redução significativa, mantendo notas entre 5 e 7. Estes resultados mostram o efeito positivo do uso da $\mathrm{AC}$ na manutenção da qualidade durante o prolongamento do período de armazenagem de pêssegos da cv. Chiripá.

\section{CONCLUSÃO}

A $\mathrm{AC}\left(1,5 \mathrm{KPa}\right.$ de $\mathrm{O}_{2}$ e $5 \mathrm{KPa}$ de $\left.\mathrm{CO}_{2}\right)$ reduziu significativamente a ocorrência de lanosidade e de escurecimento, além de preservar melhor as características organolépticas das frutas e prolongar em, no mínimo, 50\% o período de estocagem proporcionado por AR.

\section{REFERÊNCIAS BIBLIOGRÁFICAS}

BEN-ARIE, R.; SONEGO, L. Pectolytic anzyme activity involved in wolly breakdown of stored peaches. Phitochemistry, Oxford, v.19, p.2553-2555, 1980.

BRACKMANN, A.; SAQUET, A. Efeito das condições de atmosfera controlada sobre a ocorrência de degenerescência de maçã "Fuji". Scientia Agricola, Piracicaba, v.52, n.2, p.263-267, 1995

CERETA, M. Qualidade do pêssego, cv. Eldorado, armazenado em atmosfera controlada. Pelotas - RS, 1999. 46p. Dissertação (Mestrado em Agronomia) - Programa de Pós-graduação em Agronomia, Universidade Federal de Pelotas, 1999.

GOTTINARI, R.; ROMBALDI, C.V.; ARAÚJO, P.J. Frigoconservação de pêssegos da cultivar BR1. Revista Brasileira de Agrociência, Pelotas, v.4, n.1, p.47-54, 1998.

LUCHSINGER, L.E.; WALSH, C.S.; SMITH, M. Chilling injury of peach fruits during storage. Horticulturae Science, Wellesbourne, v.25, n.5, p.31-36, 1996

LURIE, S. Controlled atmosphere storage to decrease physiological disorders in nectarines. Journal Food Science and Technology, Mysore, v.27, p.507-514, 1992.

LURIE, S. Modified atmosphere storage of peaches and nectarines to reduce storage disorders. Journal Food Quality, Oxford, v.16, p.56-65, 1993 .

MANESS, N.O.; CHRIZ, D.; HEDGE, S., et al. Cell wall changes in ripening peach fruit from cultivar differing in softening rate. Acta Horticulturae, Wageningen, n.343, p.200-203, 1993.

MEDEIROS, C.A.B.; RASEIRA, M.C.B. A cultura do pessegueiro. Brasilia : EMBRAPA-SPI; Pelotas : EMBRAPA-CPACT, 1998. 251p.
MEREDITH, I.I.; ROBERTSON, A.J.; HORVAT, R. Changes in physical and chemical parameteres associated with quality and postharvest ripening of harvested peaches. Journal Agricultural and Food Chemistry, Washington, n.37, p.1210-1214, 1989.

MITCHELL, F.G.; CRISOSTO, C.H. The use cooling and cold storage to stabilize and preserve fresch stone fruits. In: VENBRELL, M.; AUDERGON, J.M. Post-harvest quality and derived products in stone-fruits. Leida : IRTA, 1995. p.125-137.

MURRAY, R.; VALENTINI, G. Storage and quality of peach fruit harvest at different stages of maturity. Acta Horticulturae, Wageningen, v.2, n.465, p.455-463, 1998.

PALMER, J.W.; WRIGHT, K.; KADER, A.A. Effect of controlled-atmosphere storage on the quality and carotenoid content of sliced persimmons and peaches. Postharvest Biology and Technology, v.10, p.89-97, 1997.

PECH, J.C.; LATCHÉ, A.; BALAGUÉ, C., et al. Postharvest physiology of climateric fruits: recent developments in the biosyntesis and action of ethylene. Science des Aliments, Paris, n.14, p.3-15, 1994.

PRATELLA, G.C.; BIONDI, G.; BASSI, R. Indici di maturazione per la raccolta: valutazione objetiva per il miglioramento qualitativo delle pesche. Rivista di Frutticoltura, Bologna, n.12, p.75-80, 1988.

PRESSEY, R.; AVANTS, J.K. Difference in polygalacturonase composition of clingstone and freestone peaches. Journal of Food Science, Chicago, v.43, p.1415-1417, 1978.

ROBERTSON, J.A.; MEREDITH, F.I.; FORBUS, W.R.J., $\boldsymbol{e t}$ al. Relatioship of quality characteristics of peachs to maturity. Journal of Food Science, Chicago, v.57, n.6, p.1401-1404, 1992.

SALVADOR, M.E.; LIZANA, L.A.; LUCSINGER, L.E., $\boldsymbol{e t}$ al Locality effect on some fruit quality parameters in peaches and nectarines. Acta Horticulturae, Wageningen, v.2, n. 465, p.447-451, 1998.

SONEGO, L.; BEN-ARIE, R.; RAYNAL, J., $\boldsymbol{e}$ t $\boldsymbol{a}$. Biochemical and physical evaluation of textural characteristics of nectarines exhibiting woolly breakdown. Postharvest Biology and Technology, Amsterdam, v.54, p.58-62, 1994.

SOUTY, M.; AUDERGON, J.M.; CHAMBROY, Y. Les critères de qualité. L'Arboriculture Frutière, Paris, n.430, p.18-24, 1990.

TAYLOR, M.A.; RABE, E.; GOOD, M.C., et al. Effect of storage regimes on pectolytic enzymes, pectic substances, internal conductivity and gel breakdown in cold storage of sungold plums. Journal Horticultural Science, Ashford, v.69, p.527-534, 1994.

TONUTTI, P.; BONGHI, C.; VIDRICH, R., et al.. Molecular and biochemical effects of anoxia, hypoxia and $\mathrm{CO}_{2}$-enriched atmosphere on Springcrest peaches. Acta Horticulturae, Waningen, v.2, n.465, p.439-446, 1998. 